\title{
Long-term outcome of patients on continuous-flow left ventricular assist device support
}

\author{
Koji Takeda, MD, PhD, ${ }^{\mathrm{a}}$ Hiroo Takayama, $\mathrm{MD}, \mathrm{PhD},{ }^{\mathrm{a}}$ Bindu Kalesan, $\mathrm{PhD}, \mathrm{MPH},{ }^{\mathrm{b}}$ Nir Uriel, MD, \\ Paolo C. Colombo, MD, ${ }^{\mathrm{c}}$ Ulrich P. Jorde, $\mathrm{MD},{ }^{\mathrm{c}}$ and Yoshifumi Naka, MD, $\mathrm{PhD}{ }^{\mathrm{a}}$
}

Objectives: Recent advances in technology and improved patient management have enabled the use of mechanical circulatory support for unexpected long-term periods. Improved long-term outcomes may facilitate the use of device therapy as an alternative to heart transplantation. However, there are scarce data about the longterm outcomes of continuous-flow left ventricular assist devices. This study sought to evaluate the long-term outcomes in patients receiving continuous-flow left ventricular assist devices.

\begin{abstract}
Methods: Between March 2004 and June 2010, 140 patients underwent continuous-flow left ventricular assist device insertion as a bridge to transplantation or a destination therapy. These patients' charts were retrospectively reviewed.

Results: The initial strategy for continuous-flow left ventricular assist device therapy was bridge to transplantation in 115 patients $(82 \%)$ and destination therapy in 25 patients $(18 \%)$. Of those, $24(17 \%)$ died on left ventricular assist device support, $94(67 \%)$ were successfully bridged to transplantation, and $1(0.71 \%)$ showed native heart recovery. Twenty-four patients $(17 \%)$ had been on continuous-flow left ventricular assist device support for more than 3 years (mean, 3.9 years; range, 3.0-7.5 years). Estimated on-device survival at 1, 3, and 5 years was $83 \%, 75 \%$, and $61 \%$, respectively. Rehospitalizations due to bleeding, cardiac events, and device-related issues were common. The freedom from rehospitalization rates at 1 and 3 years was $31 \%$ and $6.9 \%$, respectively. A total of 14 patients $(10 \%)$ required device exchange.
\end{abstract}

Conclusions: Current continuous-flow left ventricular assist devices can provide satisfactory long-term survival. However, rehospitalization is frequently required. (J Thorac Cardiovasc Surg 2014;148:1606-14)

Continuous-flow left ventricular assist devices (CF-LVADs) have become an essential therapeutic option in the standard of care for patients with end-stage heart failure. ${ }^{1}$ Clinical outcomes continue to improve through better patient selection, surgical techniques, and perioperative management. ${ }^{2,3}$ Interagency Registry for Mechanically Assisted Circulatory Support (INTERMACS) data showed that 1- and 2-year survival in patients receiving these devices reach $80 \%$ and $70 \%$, respectively. ${ }^{4}$ These favorable midterm results encourage the use of CF-LVADs both as a bridge to transplantation (BTT) and as a destination therapy (DT). The number of DT implants, as a permanent therapy, dramatically increased to more than $40 \%$ of the total LVAD implants in the United States in $2012 .^{4}$

For BTT, because of the persistent donor organ shortage, the wait time on the device support for cardiac

From the Division of Cardiothoracic Surgery, ${ }^{\mathrm{a}}$ Department of Surgery, Division of Surgery and Epidemiology, ${ }^{b}$ Department of Surgery, and Division of Cardiology, ${ }^{c}$ Department of Medicine, Columbia University Medical Center, New York, NY.

Disclosures: Ulrich Jorde and Yoshifumi Naka report consulting fees from Thoratec. All other authors have nothing to disclose with regard to commercial support.

Received for publication Dec 14, 2013; revisions received March 24, 2014; accepted for publication April 4, 2014

Address for reprints: Yoshifumi Naka, MD, PhD, 177 Fort Washington Ave, New York, NY 10032 (E-mail: yn33@cumc.columbia.edu).

$0022-5223 / \$ 36.00$

Copyright (c) 2014 by The American Association for Thoracic Surgery

http://dx.doi.org/10.1016/j.jtcvs.2014.04.009 transplantation has increased, ${ }^{5}$ and in clinical settings, we occasionally encounter patients who require unexpected long-term device support, depending on blood type, body size, and allosensitization. Consequently, long-term CF-LVAD therapy has become a more important option for the treatment of end-stage heart failure. Furthermore, with the current 1-year survival of this therapy being almost equivalent to that of cardiac transplantation, the topic on whether mechanical circulatory support could play a role of replacement for heart transplantation in patients with stage $\mathrm{D}$ heart failure is being actively discussed. ${ }^{6-8}$ However, compared with transplantation, more clinical data from long-term follow-up studies are necessary to evaluate the risks and benefits of CF-LVADs. In this study, we reviewed our single-center experience with CF-LVADs through a long-term follow-up study.

\section{METHODS}

The institutional review board of the Columbia University Medical Center approved this study. We retrospectively reviewed our experience with the CF-LVAD at the Columbia Presbyterian Medical Center between March 2004 and June 2010. During this period, 140 consecutive patients with end-stage heart failure who underwent the insertion of a CF-LVAD were included in this study. Preoperative variables that may correlate with survival were retrospectively collected for each patient. Most of these variables were selected on the basis of previous LVAD risk scores. ${ }^{9}$ The cohort consisted of 111 men with a mean age of 55 years. The initial 


\section{Abbreviations and Acronyms \\ AI $=$ aortic insufficiency \\ BTT $=$ bridge to transplantation \\ CF-LVAD = continuous-flow left ventricular assist device \\ DT $\quad=$ destination therapy \\ GI $\quad=$ gastrointestinal \\ INTERMACS $=$ Interagency Registry for Mechanically Assisted Circulatory Support \\ LVAD $\quad=$ left ventricular assist device \\ RVAD $=$ right ventricular assist device \\ TR $=$ tricuspid regurgitation}

strategy for LVAD insertion was BTT in 115 patients (82\%) and DT in 25 patients $(18 \%)$. The baseline patient characteristics are shown in Table 1.

\section{Devices and Concomitant Surgery}

LVAD support was provided by 117 HeartMate II devices (Thoratec Corp, Pleasanton, Calif), 9 VentrAssist devices (Ventracor Ltd, Chatswood, NSW, Australia), 8 DuraHeart devices (TerumoHeart, Ann Arbor, Mich), and 6 DeBakey devices (MicroMed Technology, Inc, Houston, Tex). Various concomitant procedures were performed during LVAD insertion (Table 2). Of note, 39 patients $(28 \%)$ underwent tricuspid annuloplasty (suture annuloplasty in 2, ring annuloplasty in 37 ), and 13 patients $(9.2 \%)$ underwent aortic valve repair. Two patients $(1.4 \%)$ in whom severe right ventricular failure developed in the operating room required concomitant right ventricular assist device (RVAD) insertion with a CentriMag system (Thoratec Corp).

\section{Postimplant Anticoagulation and Follow-up}

After device implantation, a standardized anticoagulation therapy with aspirin and warfarin was implemented. In patients receiving the HeartMate II device, a target international normalized ratio (INR) range was $2 \pm 0.5$. After discharge, patients' anticoagulation was managed by nursepractitioners with repeat testing frequency dictated by ease or difficulty in maintaining the patient within target INR range. Anticoagulation therapy was held in the event of bleeding and resumed once bleeding stopped. Patients were followed up at 1 week after initial discharge and then monthly unless there was an issue. There was no shared-care center during this study period. The frequency of clinic visits varied among patients depending on medical issues and the distance from a patient's home.

\section{Follow-up and Postoperative Data Collection}

The follow-up examinations were completed in June 30, 2013, and extended from 0 to 8.5 years (median, 3.6 years; interquartile range, 2.1-4.8 years). Clinical follow-up was completed in $98 \%$ of patients. To evaluate the early and late outcomes of patients during on-device support, all clinical data were collected until the patient reached 1 of 3 end points (death, transplant, or device explant for recovery). Late adverse events requiring rehospitalization included major bleeding events, such as gastrointestinal (GI) tract bleeding and significant epistaxis; device-related events, such as pump malfunction, thrombi, and infection; major cerebral events; recurrent heart failure; cardiac arrhythmia; infections not related to LVAD; and other various reasons. For patients receiving BTT, followup data after the cardiac transplantation also were collected.

To assess the time course of end-organ function after LVAD insertion, biochemical data, including blood urea nitrogen, creatinine, total and direct bilirubin, alanine aminotransferase, and aspartate aminotransferase levels, were collected at 1 and 6 months and at 1,2, and 3 years after the LVAD insertion.

Echocardiographic reports were retrospectively reviewed to assess the time course of the left ventricular dimension, aortic insufficiency (AI) severity, and tricuspid regurgitation (TR) severity. AI and TR severities were graded as none to trace, mild, mild-to-moderate, moderate, moderate-to-severe, and severe. AI and TR were considered to be significant if the grades were mild-to-moderate or greater. ${ }^{10}$ The data before; at 1 and 6 months after; and at 1, 2, and 3 years after LVAD insertion also were collected.

\section{Statistical Analysis}

The data represent frequency distributions and percentages. Continuous variables are expressed as mean \pm standard deviation and compared using 2-sample $t$ tests. Categoric variables were compared using the chi-square test. Kaplan-Meier analysis was used to calculate survival along with a log-rank $P$ value when comparing groups. Patients were censored for transplantation and native heart recovery to calculate estimated on-device survival. Patients were stratified by preoperative variables, including age, cause of heart failure, implant era (before or after approval of the HeartMate II device by the Food and Drug Administration), device type (HeartMate II or others), and the HeartMate II Risk Score profiles. ${ }^{11}$ Logistic regression was used to identify correlates of overall mortality during LVAD support. Continuous variables were dichotomized using the median value. Because of the small number of patients reviewed and lack of sufficient power, multivariate analysis was not performed. For multiple group comparison, mixed maximum likelihood regression models with log-transformed values were used.

\section{RESULTS}

\section{Early and Late Clinical Outcomes}

Early and late mortality and morbidity rates are listed in Table 3. The in-hospital mortality rate was $7.9 \%(n=11)$. Seven patients $(5.0 \%)$ had major stroke events, 2 of whom died. During hospitalization, 5 patients required delayed RVAD insertion for refractory right ventricular failure at 1 to 60 days after LVAD insertion. Of the 7 patients who eventually required RVAD support, 4 died (3 of multiorgan failure, 1 of a hemorrhagic stroke) and 3 were successfully bridged to transplantation. There were 13 late deaths during LVAD support after discharge. The leading cause of late death was device-related issues (device thrombus requiring device exchange in 1 case, sudden device malfunction in 3 cases, and operator error in battery exchange at home in 1 case). Overall, 24 patients $(17 \%)$ died on LVAD support during a mean duration of 1.2 years (range, 0.011-7.5 years). Kaplan-Meier analysis showed an estimated ondevice survival at 1,3 , and 5 years of $83 \%, 75 \%$, and $61 \%$, respectively (Figure $1, A$ ). There were no statistically significant differences in in-hospital mortality and 1-year on-device survival among patients when stratified by preoperative variables including age, cause of heart failure, implant era, device type, and the HeartMate II risk score (Table 4).

Table 5 demonstrates the risk factor analysis of overall mortality during LVAD support. Preoperative use of 
TABLE 1. Baseline characteristics

\begin{tabular}{|c|c|}
\hline Variables & $\mathbf{N}=\mathbf{1 4 0}$ \\
\hline Age, $y$, mean $\pm S D$ & $54.7 \pm 14.4 \mathrm{y}$ \\
\hline \multicolumn{2}{|l|}{ Age groups, n (\%) } \\
\hline$\leq 45 \mathrm{y}$ & $38(27.1)$ \\
\hline$>45-\leq 60 y$ & $45(32.1)$ \\
\hline$>60-\leq 80 \mathrm{y}$ & $57(40.7)$ \\
\hline Gender, male, $\mathrm{n}(\%)$ & $111(79.3)$ \\
\hline Hypertension, n (\%) & $60(42.9)$ \\
\hline Diabetes mellitus, n (\%) & $44(31.4)$ \\
\hline Hyperlipidemia, n (\%) & $35(25.0)$ \\
\hline \multicolumn{2}{|l|}{ Cause of heart failure, $n(\%)$} \\
\hline Idiopathic cardiomyopathy & $80(57.1)$ \\
\hline Ischemic cardiomyopathy & $51(36.4)$ \\
\hline Others & $9(6.4)$ \\
\hline \multicolumn{2}{|l|}{ Intention to treat, $\mathrm{n}(\%)$} \\
\hline BTT & $115(82.1)$ \\
\hline DT & $25(17.9)$ \\
\hline Inotropes support, $\mathrm{n}(\%)$ & $116(82.9)$ \\
\hline Ventilator support, $\mathrm{n}(\%)$ & $2(1.4)$ \\
\hline IABP support, $\mathrm{n}(\%)$ & $35(25.0)$ \\
\hline Mechanical circulatory support, $\mathrm{n}(\%)$ & $15(11)$ \\
\hline ICD, n $(\%)$ & $116(82.9)$ \\
\hline Reoperation, n (\%) & $37(26.4)$ \\
\hline $\mathrm{BMI}$, mean $\pm \mathrm{SD}$ & $26.1 \pm 5.2$ \\
\hline Obese, n (\%) & $28(20.0)$ \\
\hline BSA in $m^{2}$, mean $\pm S D$ & $1.93 \pm 0.2$ \\
\hline $\mathrm{BSA}>2, \mathrm{n}(\%)$ & $56(40.0)$ \\
\hline Ejection fraction $(\%)$ & $15.9 \pm 6.4$ \\
\hline Systolic blood pressure $(\mathrm{mm} \mathrm{Hg})$ & $101.9 \pm 17.6$ \\
\hline Mean PAP (mm Hg) & $35.4 \pm 9.6$ \\
\hline PCWP (mm Hg) & $24.7 \pm 8.5$ \\
\hline $\mathrm{CVP}(\mathrm{mm} \mathrm{Hg})$ & $11.6 \pm 6.4$ \\
\hline $\mathrm{CO}(\mathrm{L} / \mathrm{min})$ & $3.0 \pm 0.95$ \\
\hline PVR (Wood units) & $4.1 \pm 2.7$ \\
\hline Sodium (mmol/L) & $133 \pm 4.5$ \\
\hline BUN (mg/dL) & $34 \pm 17$ \\
\hline Creatinine (mg/dL) & $1.5 \pm 0.64$ \\
\hline Total protein $(\mathrm{g} / \mathrm{dL})$ & $6.7 \pm 0.85$ \\
\hline Albumin (g/dL) & $3.6 \pm 0.55$ \\
\hline ALT (IU/L) & $41 \pm 64$ \\
\hline AST (IU/L) & $67 \pm 199$ \\
\hline Total bilirubin (mg/dL) & $1.5 \pm 0.90$ \\
\hline Direct bilirubin (mg/dL) & $0.48 \pm 0.38$ \\
\hline $\mathrm{WBC}(\times 1000 / \mathrm{mL})$ & $8.8 \pm 3.5$ \\
\hline Hemoglobin (g/dL) & $11 \pm 2.0$ \\
\hline $\operatorname{HCT}(\%)$ & $34 \pm 5.7$ \\
\hline Platelets $(\times 1000 / \mathrm{mL})$ & $216 \pm 70$ \\
\hline INR & $1.3 \pm 0.37$ \\
\hline
\end{tabular}

$\overline{A L T \text {, Alanine aminotransferase; } A S T \text {, aspartate aminotransferase; } B M I \text {, body mass }}$ index; $B S A$, body surface area; $B T T$, bridge to transplantation; $B U N$, blood urea nitrogen; $C O$, cardiac output; $C V P$, central venous pressure; $D T$, destination therapy; $H C T$, hematocrit; $I A B P$, intra-aortic balloon pump; $I C D$, implantable cardioverterdefibrillator; $I N R$, international normalized ratio; $P A P$, pulmonary artery pressure; $P C W P$, pulmonary capillary wedge pressure; $P V R$, pulmonary vascular resistance; $S D$, standard deviation; $W B C$, white blood cells.

intra-aortic balloon pump, baseline hemoglobin, hematocrit, and albumin level were significant predictors of mortality.
TABLE 2. Concomitant surgical procedures

\begin{tabular}{lc}
\hline Tricuspid valve repair, $\mathrm{n}(\%)$ & $39(28)$ \\
Aortic valve repair, $\mathrm{n}(\%)$ & $13(9.3)$ \\
Aortic valve replacement, $\mathrm{n}(\%)$ & $1(0.71)$ \\
Closure of prosthetic aortic valve, $\mathrm{n}(\%)$ & $2(1.4)$ \\
Mitral valve repair, $\mathrm{n}(\%)$ & $3(2.1)$ \\
PFO closure, n (\%) & $9(6.4)$ \\
Left ventricular aneurysmectomy, n (\%) & $2(1.4)$ \\
Concomitant RVAD insertion, $\mathrm{n}(\%)$ & $2(1.4)$ \\
\hline
\end{tabular}

$P F O$, Patent foramen ovale; $R V A D$, right ventricular assist device.

A total of 84 patients $(60 \%)$ underwent 169 rehospitalizations (209 patient-years) related to adverse events (Table 3). The leading causes of readmission included major bleeding events, recurrent heart failure, device thrombus/malfunction, device infection, and cardiac arrhythmia. The adverse event rates (number of events/ patient-year) are listed in Table 3. The freedom from rehospitalization rates for hospital survivors at 1 and 3 years were $31 \%$ and $6.9 \%$, respectively (Figure $1, B$ ).

TABLE 3. Early and late mortality and morbidity during continuousflow left ventricular assist device support

\begin{tabular}{|c|c|}
\hline In-hospital mortality, n (\%) & $11(7.9)$ \\
\hline Multiorgan failure & $5(3.6)$ \\
\hline Stroke & $2(1.4)$ \\
\hline Miscellaneous cause & $2(1.4)$ \\
\hline Sepsis & $1(0.71)$ \\
\hline Heparin-induced thrombocytopenia & $1(0.71)$ \\
\hline \multicolumn{2}{|l|}{ In-hospital morbidity, n (\%) } \\
\hline Cardiac arrhythmia & $33(24)$ \\
\hline Reoperation for bleeding & $22(16)$ \\
\hline Acute renal failure requiring dialysis & $16(11)$ \\
\hline Sepsis & $7(5.0)$ \\
\hline Stroke & $7(5.0)$ \\
\hline RVAD use & $7(5.0)$ \\
\hline Venovenous ECMO use & $1(0.71)$ \\
\hline Late mortality, n (\%) & $13(9.3)$ \\
\hline Device-related & $5(3.6)$ \\
\hline Stroke & $1(0.71)$ \\
\hline Respiratory failure & $1(0.71)$ \\
\hline Sepsis & $1(0.71)$ \\
\hline Miscellaneous cause & $5(3.6)$ \\
\hline \multicolumn{2}{|l|}{ Rehospitalization, no. of events (per patient-y) } \\
\hline Bleeding & $30(0.14)$ \\
\hline Device thrombus/malfunction & $19(0.091)$ \\
\hline Device infection & $21(0.10)$ \\
\hline Heart failure & $26(0.12)$ \\
\hline Cardiac arrhythmia & $16(0.077)$ \\
\hline Stroke & $9(0.043)$ \\
\hline LVAD nonrelated infection & $11(0.053)$ \\
\hline Device alarm without mechanical abnormality & $12(0.057)$ \\
\hline TIA or syncope & $8(0.038)$ \\
\hline Psychiatric issues & $3(0.014)$ \\
\hline Others & $14(0.067)$ \\
\hline
\end{tabular}

$E C M O$, Extracorporeal membrane oxygenation; $L V A D$, left ventricular assist device; $R V A D$, right ventricular assist device; $T I A$, transient ischemic attack. 

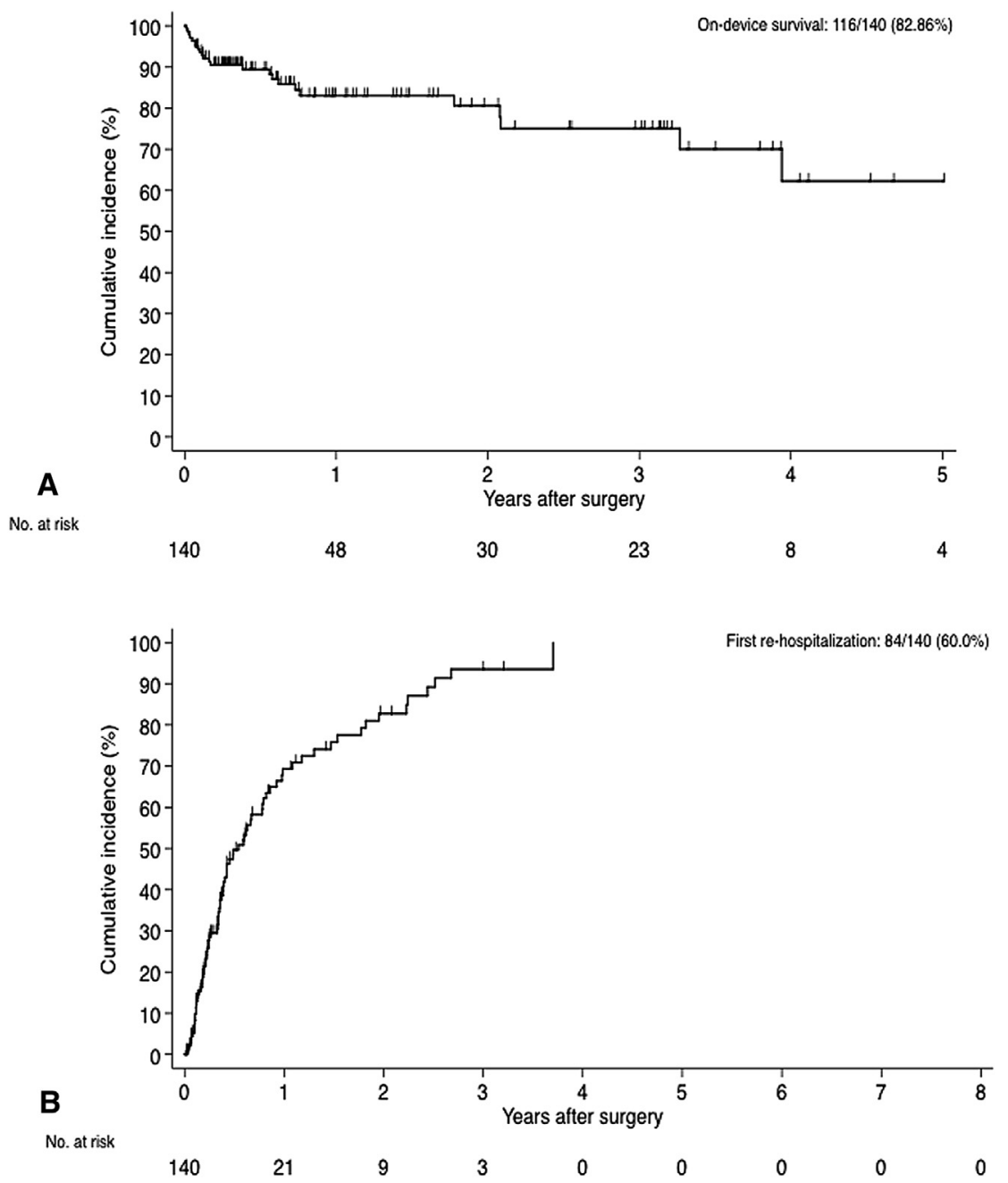

FIGURE 1. A, On-device survival of the entire cohort. B, Freedom from rehospitalization rate for any reason.

A total of 14 patients (10\%) (13 HeartMate II pumps and 1 DeBakey pump) underwent pump replacement because of device thrombosis $(\mathrm{n}=5)$, driveline fracture $(\mathrm{n}=3)$, infection $(\mathrm{n}=2)$, and other reasons $(\mathrm{n}=4)$. The median time to pump replacement was 1.6 years (range, 0.019-4.9 years). Pump replacement was performed through a median full sternotomy $(\mathrm{n}=9)$ or subcostal incision $(\mathrm{n}=5) .{ }^{12}$ There were 2 in-hospital deaths in the full-sternotomy group. The rates of freedom from pump replacement at 1,2,3, and 5 years were $94 \%, 83 \%, 69 \%$, and $51 \%$, respectively.

\section{Patients on Device Support More Than 3 Years}

A total of 24 patients (17\%) had been on LVAD support for more than 3 years (mean, 3.9 years; range, 3.0-7.5 years). The devices used included the HeartMate II in 23 patients and the VentrAssist in 1 patient. Three patients were bridged to transplantation, and 5 patients were listed for transplantation. There were 63 major adverse events in 22 patients $(92 \%)$. Eighteen patients $(75 \%)$ underwent multiple readmissions. The annual rehospitalization rate was 2.3 per patient-year for the first year (17 events in 12 patients), 1.8 per patient-year for the second year (17 events in 14 patients), 2.3 per patient-year for the third year (18 events in 11 patients), and 1.5 per patient-year during the 3-year follow-up period (11 events in 8 patients).

\section{Bridge to Transplantation, Recovery, and Destination Therapy}

A total of 94 patients $(67 \%)$ were successfully bridged to transplantation after a mean of 0.76 years of LVAD support (range, 0.074-3.9 years). The initial strategies were BTT in 92 patients and DT in 2 patients. The BTT rate in patients who were initially eligible for transplantation was $79 \%$. The in-hospital mortality rate after transplantation in these BTT cases was $5.3 \%(n=5)$. The actuarial survivals after transplantation at 1,3 , and 5 years were $88 \%, 86 \%$, and $80 \%$, respectively. One patient with doxorubicin-induced cardiomyopathy was successfully bridged to recovery after 
TABLE 4. In-hospital mortality and on-device survival at 1 year stratified by preoperative variables

\begin{tabular}{|c|c|c|c|c|c|}
\hline Variable & No. of patients $(\%)$ & In-hospital mortality (\%) & $P$ value & Survival at 1 y $(\%)$ & $P$ value \\
\hline \multicolumn{6}{|l|}{ Age } \\
\hline$\leq 45 \mathrm{y}$ & $38(27.1)$ & 7.89 & & 86.0 & \\
\hline$>45-\leq 60$ y & $45(32.1)$ & 8.89 & & 79.6 & \\
\hline$>60-\leq 80$ y & $57(40.7)$ & 7.02 & .94 & 83.8 & .91 \\
\hline \multicolumn{6}{|l|}{ Cause } \\
\hline Idiopathic cardiomyopathy & $80(57.1)$ & 7.50 & & 82.3 & \\
\hline Ischemic cardiomyopathy & $51(36.4)$ & 7.84 & .94 & 81.2 & .59 \\
\hline \multicolumn{6}{|l|}{ Implant era } \\
\hline March 2004 to April 2008 & $47(33.6)$ & 10.6 & & 71.1 & \\
\hline May 2008 to June 2010 & $93(66.4)$ & 6.45 & .33 & 86.9 & .19 \\
\hline \multicolumn{6}{|l|}{ Device type } \\
\hline HeartMate II (Thoratec Corp, Pleasanton, Calif) & $117(83.6)$ & 6.84 & & 83.3 & \\
\hline Others & $23(16.4)$ & 13.0 & .31 & 77.9 & .86 \\
\hline \multicolumn{6}{|l|}{$\mathrm{HMRS} \dagger$} \\
\hline Low risk $(\leq 1.58)$ & $76(54.3)$ & 5.26 & & 87.8 & \\
\hline Medium risk (1.58-2.48) & $48(34.3)$ & 8.33 & & 77.4 & \\
\hline High risk $(>2.48)$ & $16(11.4)$ & 18.8 & .19 & 72.2 & .22 \\
\hline
\end{tabular}

0.91 years of LVAD support. However, this patient experienced recurrent stage D heart failure 6 months after device removal, required LVAD reinsertion, and was eventually bridged to transplantation. The patients receiving DT received LVAD support for an average of 3.2 years (range, 0.010-7.5 years). Nine patients receiving DT died during the support period. The on-device survival was similar between the BTT and DT groups $(P=.79)$.

\section{End-Organ Function During Continuous-Flow Left Ventricular Assist Device Support}

Table 6 demonstrates the time course of hepatic and renal function before and after CF-LVAD insertion. End-organ function was restored 1 month after initiation of support. These improvements had been sustained throughout the support period except for creatinine level. Three-year longitudinal data were available in 19 patients. Mean creatinine level in this group was $1.5 \pm 0.50$ at baseline, which decreased to $1.1 \pm 0.39$ at 1 month $(P=.050$, vs baseline $)$ and increased to $1.4 \pm 0.48$ at 3 years $(P=.61$, vs baseline $)$.

\section{Change of Left Ventricular Dimension, Aortic Insufficiency, and Tricuspid Regurgitation}

Figure 2 shows the serial change of left ventricular diastolic dimension during LVAD support. Left ventricular diastolic dimension significantly decreased from $69 \pm 11 \mathrm{~mm}$ to $58 \pm 11 \mathrm{~mm}$ at 1 month after LVAD support $(P<.0001)$; thereafter, the dimension has remained stable.

The ratio of significant $\mathrm{AI}$ increased over time (Figure 3, $A$ ). One third of patients had more than mild-to-moderate AI in the late period. In 13 patients who underwent concomitant aortic valve repair, 1 had recurrent severe
AI at 1 month after initial surgery. Others had less than mild AI during a mean follow-up of 1.4 years (range, 0.11-3.5 years).

Of the 15 patients who required readmission because of heart failure, 6 had typical right-sided heart failure symptoms (eg, leg edema) and 8 had typical left-sided symptoms (eg, shortness of breath). Biventricular failure symptoms developed in 1 patient. Seven of 17 patients $(41 \%)$ with significant $\mathrm{AI}$ at any postoperative point underwent rehospitalization because of left-sided heart failure compared with 2 of 113 patients $(1.8 \%)$ who did not have significant AI $(P<.0001)$. The ratio of significant TR decreased from $45 \%$ to $22 \%$ at 1 month after LVAD insertion and remained at approximately $20 \%$ during follow-up (Figure 3, B). In those undergoing concomitant tricuspid surgery $(\mathrm{n}=39)$, 3 patients had recurrent TR (mild-to-moderate in 2, severe in 1) during follow-up. TR severity was consistently less than mild in all the other patients. Similar to those with AI, 5 of 33 patients $(15 \%)$ with significant TR at any postoperative point were rehospitalized because of right-sided heart failure compared with 2 of 97 patients $(2.0 \%)$ who did not have significant TR $(P=.012)$.

\section{DISCUSSION}

This study demonstrated our single-center experience of CF-LVADs as a long-term therapy for patients with heart failure. Satisfactory long-term on-device survivals of $83 \%$ at 1 year, $75 \%$ at 3 years, and $61 \%$ at 5 years were obtained. Because the prospective, randomized clinical trial demonstrated a significant improvement in survival and device durability with the CF-LVAD compared with the pulsatile-flow LVAD, use of the CF pump has rapidly 
TABLE 5. Predictors of on-device mortality, $N=140$

\begin{tabular}{|c|c|c|c|c|}
\hline \multirow[b]{2}{*}{ Variables } & \multicolumn{2}{|c|}{ On-device mortality, n ( $\%)$} & \multirow[b]{2}{*}{ OR, $95 \%$ CI } & \multirow[b]{2}{*}{$P$ value } \\
\hline & Yes (24) & No (116) & & \\
\hline Age groups (Ref: $\leq 45$ y) & & & & .93 \\
\hline$>45-\leq 60 y$ & $8(33.3)$ & 37 (31.9) & $1.02(0.35-2.93)$ & \\
\hline$>60-\leq 80 y$ & $10(41.7)$ & $47(40.5)$ & $0.86(0.31-2.41)$ & \\
\hline \multicolumn{5}{|l|}{ Gender (Ref: female) } \\
\hline Male & $19(79.2)$ & $92(79.3)$ & $1.24(0.46-3.35)$ & .67 \\
\hline Obesity (BMI $>30 \mathrm{~kg} / \mathrm{m}^{2}$ ) (Ref: No) & $7(29.2)$ & $21(18.1)$ & $1.55(0.64-3.73)$ & .33 \\
\hline Hypertension (Ref: No) & $10(41.7)$ & $50(43.1)$ & $0.79(0.35-1.79)$ & .57 \\
\hline Diabetes (Ref: No) & $7(29.2)$ & 37 (31.9) & $0.74(0.31-1.79)$ & .50 \\
\hline Hyperlipidemia (Ref: No) & $4(16.7)$ & $31(26.7)$ & $0.58(0.20-1.71)$ & .32 \\
\hline Cause of heart failure (Ref: idiopathic cardiomyopathy) & & & & .85 \\
\hline Ischemic cardiomyopathy & $8(33.3)$ & $43(37.1)$ & $0.78(0.33-1.88)$ & \\
\hline Others & $2(8.3)$ & $7(6.0)$ & $1.06(0.24-4.71)$ & \\
\hline DT (Ref: BTT) & $9(37.5)$ & $16(13.8)$ & $1.57(0.65-3.81)$ & .32 \\
\hline Inotropes support (Ref: No) & $16(66.7)$ & $100(86.2)$ & $0.47(0.20-1.11)$ & .087 \\
\hline IABP support (Ref: No) & $9(37.5)$ & $26(22.4)$ & $2.77(1.18-6.48)$ & .019 \\
\hline ICD (Ref: No) & $19(79.2)$ & 97 (83.6) & $0.78(0.29-2.09)$ & .62 \\
\hline Reoperation (Ref: No) & $9(37.5)$ & $28(24.1)$ & $1.26(0.54-2.91)$ & .59 \\
\hline Ejection fraction $\geq 15(\%)$ & $14(58.3)$ & $69(59.5)$ & $1.04(0.48-2.42)$ & .86 \\
\hline Systolic blood pressure $\geq 101(\mathrm{~mm} \mathrm{Hg})$ & $8(33.3)$ & $49(42.2)$ & $0.68(0.29-1.59)$ & .37 \\
\hline $\mathrm{CVP} \geq 11(\mathrm{~mm} \mathrm{Hg})$ & $5(20.8)$ & $26(22.4)$ & $0.96(0.36-2.58)$ & .94 \\
\hline $\mathrm{PAP} \geq 36(\mathrm{~mm} \mathrm{Hg})$ & $9(37.5)$ & $53(45.7)$ & $0.75(0.33-1.72)$ & .50 \\
\hline PCWP $\geq 25(\mathrm{~mm} \mathrm{Hg})$ & $10(41.7)$ & $55(47.4)$ & $0.84(0.37-1.89)$ & .67 \\
\hline $\mathrm{CO} \geq 3.0(\mathrm{~L} / \mathrm{min})$ & $14(58.3)$ & $48(41.4)$ & $1.59(0.70-3.59)$ & .26 \\
\hline PVR $\geq 3.5$ (Wood units) & $9(37.5)$ & $52(44.8)$ & $0.80(0.35-1.83)$ & .59 \\
\hline $\mathrm{WBC} \geq 8.2(\times 1000 / \mathrm{mL})$ & $14(58.3)$ & $57(49.1)$ & $1.64(0.72-3.71)$ & .23 \\
\hline Hemoglobin $\geq 11$ (g/dL) & $8(33.3)$ & $63(54.3)$ & $0.38(0.16-0.90)$ & .028 \\
\hline $\mathrm{HCT} \geq 34(\%)$ & $8(33.3)$ & $62(53.5)$ & $0.38(0.15-0.91)$ & .030 \\
\hline Platelets $\geq 211(\times 1000 / \mathrm{mL})$ & $11(45.8)$ & $58(50.0)$ & $1.02(0.45-2.30)$ & .96 \\
\hline Sodium $\geq 134(\mathrm{mmol} / \mathrm{L})$ & $11(45.8)$ & $63(54.3)$ & $0.68(0.31-1.53)$ & .36 \\
\hline $\mathrm{BUN} \geq 31.5(\mathrm{mg} / \mathrm{dL})$ & $14(58.3)$ & $55(47.4)$ & $1.38(0.60-3.11)$ & .44 \\
\hline Creatinine $\geq 1.5(\mathrm{mg} / \mathrm{dL})$ & $16(66.7)$ & $54(46.6)$ & $2.05(0.88-4.80)$ & .098 \\
\hline Total protein $\geq 6.7$ (g/dL) & $12(50.0)$ & $60(51.7)$ & $0.85(0.38-1.89)$ & .68 \\
\hline Albumin $\geq 3.6(\mathrm{~g} / \mathrm{dL})$ & $9(37.5)$ & $71(61.2)$ & $0.37(0.16-0.85)$ & .019 \\
\hline Total bilirubin $\geq 1.15(\mathrm{mg} / \mathrm{dL})$ & $13(54.2)$ & $56(48.3)$ & $1.45(0.65-3.26)$ & .37 \\
\hline Direct bilirubin $\geq 0.35(\mathrm{mg} / \mathrm{dL})$ & $14(58.3)$ & $54(46.6)$ & $1.80(0.79-4.07)$ & .16 \\
\hline $\mathrm{AST} \geq 25$ (IU/L) & $11(45.8)$ & $60(51.7)$ & $0.75(0.34-1.67)$ & .48 \\
\hline ALT $\geq 23(\mathrm{IU} / \mathrm{L})$ & $10(41.7)$ & $62(53.5)$ & $0.70(0.31-1.58)$ & .39 \\
\hline INR $\geq 1.2$ & $12(50.0)$ & $54(46.6)$ & $1.29(0.58-2.89)$ & .53 \\
\hline
\end{tabular}

$A L T$, Alanine aminotransferase; $A S T$, aspartate aminotransferase; $B M I$, body mass index; $B T T$, bridge to transplantation; $B U N$, blood urea nitrogen; $C I$, confidence interval; $C O$, cardiac output; $C V P$, central venous pressure; $D T$, destination therapy; $H C T$, hematocrit; $I A B P$, intra-aortic balloon pump; $I C D$, implantable cardioverter-defibrillator; $I N R$, international normalized ratio; $O R$, odds ratio; $P A P$, pulmonary artery pressure; $P C W P$, pulmonary capillary wedge pressure; $P V R$, pulmonary vascular resistance; $R e f$, reference; $W B C$, white blood cells.

increased. ${ }^{1-4}$ After the increase in clinical experience with these devices, improvements in outcomes have been reported. The original HeartMate II BTT trial including patients post-transplant demonstrated a 1-year overall survival of $68 \%,{ }^{1}$ which has improved to $85 \%$ according to more recent data. ${ }^{3}$ Even more recently, the ADVANCE Bridge to Transplant Trial with another CF pump (HeartWare ventricular assist device, HeartWare Inc, Framingham, Mass) showed $94 \%$ on-device survival at 180 days after implantation or explantation to receive a cardiac transplantation or for recovery. ${ }^{13}$
In the HeartMate II DT trial, 2-year survival improved from $58 \%$ in the early era to $63 \%$ in the later era. ${ }^{14}$ Thus, in the current era, excellent early and midterm survivals can be expected with CF pump technology. However, longer-term data over 2 years remain limited. The current data shed light on the paucity of long-term data for CF-LVAD therapy.

From the current results, better long-term survival can be largely expected from postdischarge patient treatment because most patients on long-term device support underwent rehospitalization. The leading causes of readmission 
TABLE 6. Time course of hepatic and renal function during continuous-flow left ventricular assist device support

\begin{tabular}{|c|c|c|c|c|c|c|c|}
\hline & Baseline & $1 \mathrm{mo}$ & $6 \mathrm{mo}$ & $1 \mathbf{y}$ & $2 y$ & $3 \mathbf{y}$ & \\
\hline & $n=141$ & $\mathbf{n}=\mathbf{1 3 0}$ & $\mathbf{n}=\mathbf{7 7}$ & $\mathbf{n}=\mathbf{5 1}$ & $\mathbf{n}=\mathbf{2 8}$ & $\mathbf{n}=\mathbf{1 9}$ & $P$ value \\
\hline AST (IU/L) & $41 \pm 64$ & $27 \pm 13$ & $34 \pm 15$ & $35 \pm 17$ & $27 \pm 10$ & $27 \pm 9.1$ & .81 \\
\hline ALT (IU/L) & $67 \pm 199$ & $22 \pm 15$ & $31 \pm 17$ & $31 \pm 26$ & $21 \pm 8.9$ & $20 \pm 11$ & .014 \\
\hline Total bilirubin (mg/dL) & $1.5 \pm 0.90$ & $0.96 \pm 0.65$ & $0.91 \pm 0.48$ & $0.85 \pm 0.48$ & $0.83 \pm 0.33$ & $0.71 \pm 0.27$ & $<.0001$ \\
\hline BUN (mg/dL) & $34 \pm 17$ & $22 \pm 11$ & $27 \pm 12$ & $27 \pm 12$ & $23 \pm 9.6$ & $24 \pm 11$ & .012 \\
\hline Creatinine $(\mathrm{mg} / \mathrm{dL})$ & $1.5 \pm 0.62$ & $1.1 \pm 0.45$ & $1.3 \pm 0.48$ & $1.4 \pm 0.47$ & $1.3 \pm 0.36$ & $1.4 \pm 0.48$ & .17 \\
\hline
\end{tabular}

$A L T$, Alanine aminotransferase; $A S T$, aspartate aminotransferase; $B U N$, blood urea nitrogen.

were bleeding, cardiac-related causes (heart failure or arrhythmia), and device-related morbidities. These issues in the chronic phase are somewhat unique compared with those in the acute phase such as stroke, sepsis, and organ failure. Hashin and colleagues ${ }^{15}$ recently reported similar findings. They reported a readmission rate of 1.64 per patient-year with a mean follow-up of 1.4 years. Of note, the readmission rate decreased from 2.0 per patient-year to 1.2 per patient-year during the first 6 months and then stabilized. In our specific population of patients who had been on device support for a mean of 4 years, there was a peak in the rehospitalization rate in the first year and another peak in the third year (2.3 per patient-year for both years). These differences may result from our longer follow-up period. In the third year, 5 of 18 events resulted from device-related issues, including 3 pump replacements.

GI tract bleeding and epistaxis have emerged as a major source of morbidity in patients with CF-LVADs. ${ }^{16}$ Possible mechanisms with obligate device anticoagulation include acquired von Willebrand disease, ${ }^{17}$ GI tract arteriovenous malformations associated with reduced pulsatility, ${ }^{18}$ and impaired platelet aggregation. ${ }^{19}$ The management of bleeding issues is inextricably linked to the risk of thromboembolic events. However, considering the individual risks and benefits, careful adjustment of anticoagulation and antiplatelet therapy seems to be the only method for treating

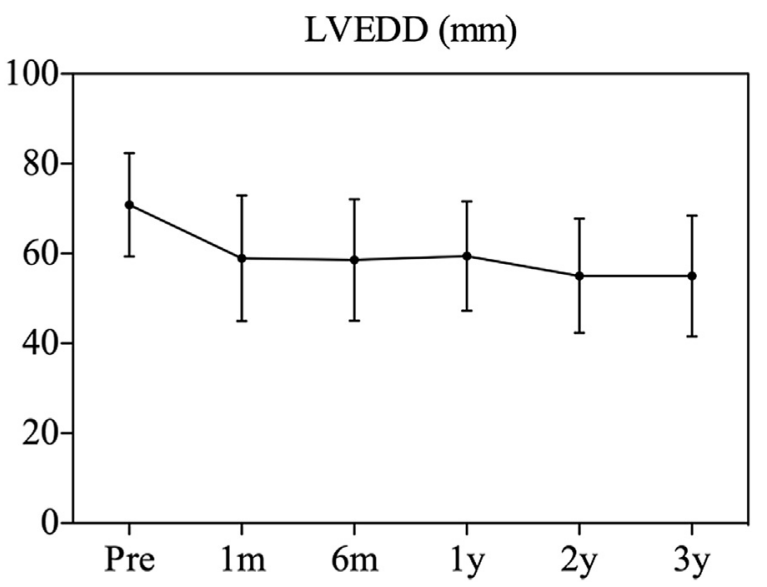

FIGURE 2. Serial change of left ventricular diastolic dimension during CF-LVAD support. LVEDD, Left ventricular end-diastolic diameter. this complication. Further understanding of the mechanisms underlying bleeding and novel strategies such as new anticoagulant drugs will play an important role in the long-term management of CF-LVAD therapy.

The development of de novo AI during CF-LVAD support has been reported. ${ }^{10,20}$ Consistent with those reports, the current study demonstrated worsening of AI over time. At 2 to 3 years of follow-up, approximately $30 \%$ of patients had significant AI. Significant AI can cause the recycling of blood flow from the LVAD outflow graft into the left ventricle and result in decreased forward cardiac output, inadequate ventricular unloading, and increased pump work. Toda and colleagues ${ }^{21}$ reported that de novo AI had

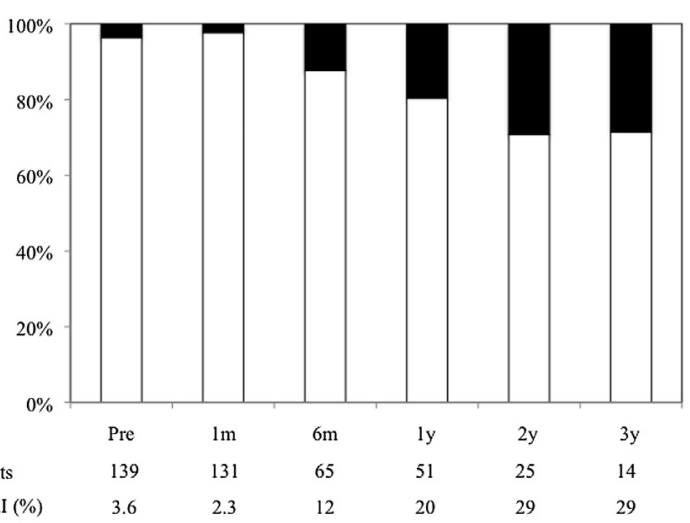

1612 The Journal of Thoracic and Cardiovascular Surgery • October 2014 
a significant negative impact on survival during long-term pulsatile LVAD support. In our study, patients with extensive AI during long-term CF-LVAD support were at significant risk of recurrent left-sided heart failure. In our current practice, to prevent the future development of $\mathrm{AI}$, we aggressively perform the concomitant aortic valve procedure for cases with more than mild-to-moderate AI. ${ }^{22}$ Although recurrence developed in 1 patient who had preoperative severe $\mathrm{AI}$, the outcome of concomitant aortic valve plasty seems satisfactory. With regard to TR, approximately $20 \%$ of patients constantly had a significant degree of regurgitation. The presence of significant TR usually reflects right ventricular dysfunction or volume overload status. In our study, as noted with AI, TR also had a significant negative impact on late functional status. Piacentino and colleagues $^{23}$ demonstrated that the use of a concomitant tricuspid valve procedure can efficiently eliminate TR and prevent postoperative right ventricular heart failure. Therefore, concomitant aortic and tricuspid valve plasty or replacement depending on valve pathology should be considered for patients who need long-term CF-LVAD support.

Cardiac arrhythmia, especially ventricular arrhythmia, also was a common issue in the early and late periods. Although such arrhythmia may not be lethal in the presence of LVAD, it could put patients at risk of right ventricular failure. In addition to implantable cardioverter-defibrillator therapy, interventions aimed at minimizing the risk of recurrent arrhythmias, such as antiarrhythmic medication use, catheter ablation, and even intraoperative cryoablation, may be considered. ${ }^{24,25}$

In our study, device-related events were the leading cause of late death and morbidity. Fourteen patients $(10 \%)$ required device exchange at a median time of 1.6 years. Most recently, Moazami and colleagues ${ }^{26}$ reported pump exchange data from multicenter clinical trials. A total of $72(6.4 \%)$ of 1128 patients with a HeartMate II device required an exchange at a median time of 428 days after the initial implantation. The freedom from device replacement rate at 2 years was $87 \%$ before 2007 and $92 \%$ since 2007. In our study, the freedom from device exchange rate was $94 \%$ at 1 year, $83 \%$ at 2 years, $69 \%$ at 3 years, and $51 \%$ at 5 years. Although advances in technology certainly improve device durability, prolonged device use will inevitably result in mechanical issues. Our novel echocardiography ramp test is helpful to detect device malfunction in patients with CF-LVADs. ${ }^{27}$ Routine monitoring may reduce the incidence of late mortality and morbidity associated with sudden device malfunction. In regard to the surgical approach to device exchange, the subcostal approach is currently our preferred method because it is less invasive and has better outcomes. ${ }^{12,28}$

Another interesting aspect of this study is the long-term effect of continuous blood flow on renal and hepatic function. Studies have demonstrated no deleterious effects of reduced pulsatility on end-organ function in the 6- to 15 -month time period. ${ }^{29,30}$ Our findings confirm that the use of CF-LVADs can improve renal and hepatic function with longer-term follow-up. Only the creatinine level returned to the baseline value at 3 years. This trend might be explained by the repeated rehospitalizations requiring renally toxic medications, such as antibiotics and overdoses of diuretics. In our cohort, 1 patient receiving DT in whom renal failure developed required dialysis after prolonged antibiotic therapy for bacteremia. A further detailed study would be desirable to evaluate the long-term effect of CF-LVAD therapy on renal function.

Active discussion continues about the long-term use of CF-LVADs as an alternative to cardiac transplantation. The current post-transplant survivals at 1, 2, 5, and 10 years are approximately $90 \%, 80 \%, 70 \%$, and $50 \%$, respectively. ${ }^{31}$ Kirklin and colleagues ${ }^{6}$ recently summarized a large body of data from the INTERMACS Registry including 1160 patients receiving DT. After excluding patients requiring biventricular support and notable preoperative risk factors such as cardiogenic shock, patients receiving DT within this cohort had 1- and 2-year survivals of $88 \%$ and $80 \%$, respectively, with CF-LVADs. ${ }^{6}$ Our ondevice survival data also support the utility of CF-LVADs. Excellent post-transplant survival in patients receiving BTT can reduce the need for cardiac transplantation as a first-line replacement therapy. However, it is too premature to make conclusions about survival comparisons because of the lack of head-to-head comparative data. Furthermore, considering the frequent readmissions in this population, patient survival, quality of life, and health care costs should be taken into account before conclusions are drawn.

\section{Study Limitations}

First, the study was a retrospective analysis of a singlecenter experience. Although recent multicenter studies have shown evidence of the excellent CF-LVAD outcomes, the strength of the present single-center study was the large number of patients receiving CF-LVADs with consistent strategies in patient selection, operative procedure, and postoperative management. Furthermore, the present study included detailed analyses of serial echocardiography, endorgan function, and clinical follow-up outcomes that could not be addressed in the multicenter studies. Second, the number of patients receiving DT who were deemed to be candidates for long-term support was low. For patients receiving BTT, a rather short support time was expected as an initial intent. However, the constant shortage of available donors has resulted in an increasing number of patients receiving BTT with longer waiting times on CF-LVAD support. In the present cohort, 30 of 115 patients receiving BTT (26\%) required CF-LVAD support for more than 1 year. Furthermore, INTERMACS registry data demonstrate that 
patients often have long durations of support regardless of initial implant strategy, and strategies often change over time. ${ }^{32}$ Thus, in the current continuous-flow era, device intent of BTT and DT becomes less representative of the clinical circumstances. Third, the number of readmissions to other hospitals was not recorded; thus, the overall incidence may be underestimated. Finally, various devices were included in this study. However, we intended to reflect the actual conditions prevailing in CF-LVAD therapy because various technologies emerge rapidly.

\section{CONCLUSIONS}

Current CF-LVADs provide satisfactory long-term survival, although rehospitalization for specific reasons in this population was common. With appropriate device and patient management, acceptable survivals at more than 3 years can be expected in select patients.

\section{References}

1. Slaughter MS, Rogers JG, Milano CA, et al. Advanced heart failure treated with continuous-flow left ventricular assist device. N Engl J Med. 2009;361:2241-51.

2. Pagani FD, Miller LW, Russell SD, et al. Extended mechanical circulatory support with a continuous-flow rotary left ventricular assist device. J Am Coll Cardiol. 2009;54:312-21.

3. Starling RC, Naka Y, Boyle AJ, et al. Results of the post-U.S. Food and Drug Administration-approval study with a continuous flow left ventricular assist device as a bridge to heart transplantation: a prospective study using the INTERMACS (Interagency Registry for Mechanically Assisted Circulatory Support). J Am Coll Cardiol. 2011;57:1890-8.

4. Kirklin JK, Naftel DC, Kormos RL, et al. Fifth INTERMACS annual report: risk factor analysis from more than 6,000 mechanical circulatory support patients. J Heart Lung Transplant. 2013;32:141-56.

5. Uriel N, Jorde UP, Woo Pak S, et al. Impact of long term left ventricular assist device therapy on donor allocation in cardiac transplantation. J Heart Lung Transplant. 2013;32:188-95.

6. Kirklin JK, Naftel DC, Pagani FD, et al. Long-term mechanical circulatory support (destination therapy): on track to compete with heart transplantation? J Thorac Cardiovasc Surg. 2012;144:584-603.

7. Mulloy DP, Bhamidipati CM, Stone ML, et al. Orthotopic heart transplant versus left ventricular assist device: a national comparison of cost and survival. J Thorac Cardiovasc Surg. 2013;145:566-74.

8. Garbade J, Barten MJ, Bittner HB, et al. Heart transplantation and left ventricular assist device therapy: two comparable options in end-stage heart failure? Clin Cardiol. 2013;36:378-82.

9. Lietz K, Long JW, Kfoury AG, et al. Outcomes of left ventricular assist device implantation as destination therapy in the post-REMATCH era: implications for patient selection. Circulation. 2007;116:497-505.

10. Pak SW, Uriel N, Takayama H, et al. Prevalence of de novo aortic insufficiency during long-term support with left ventricular assist devices. J Heart Lung Transplant. 2010;29:1172-6.

11. Cowger J, Sundareswaran K, Rogers JG, et al. Predicting survival in patients receiving continuous flow left ventricular assist devices: the HeartMate II risk score. J Am Coll Cardiol. 2013;61:313-21.

12. Nishimura T, Ota T, Takayama H, et al. Subcostal to rib-cross incision for HeartMate II explantation: a case report. J Thorac Cardiovasc Surg. 2013;145:e16-7.
13. Aaronson KD, Slaughter MS, Miller LW, et al. Use of an intrapericardial, continuous-flow, centrifugal pump in patients awaiting heart transplantation. Circulation. 2012;125:3191-200.

14. Park SJ, Milano CA, Tatooles AJ, et al. Outcomes in advanced heart failure patients with left ventricular assist devices for destination therapy. Circ Heart Fail. 2012;5:241-8.

15. Hashi T, Marmor Y, Kremers W, et al. Readmissions after implantation of axial flow left ventricular assist device. J Am Coll Cardiol. 2013;61:153-63.

16. Suarez J, Patel CB, Felker GM, et al. Mechanisms of bleeding and approach to patients with axial-flow left ventricular assist devices. Circ Heart Fail. 2011;4: 779-84.

17. Uriel N, Pak SW, Jorde UP, et al. Acquired von Willebrand syndrome after continuous-flow mechanical device support contributes to a high prevalence of bleeding during long-term support and at the time of transplantation. J Am Coll Cardiol. 2010;56:1207-13.

18. Wever-Pinzon O, Selzman CH, Drakos SG, et al. Pulsatility and the risk of nonsurgical bleeding in patients supported with the continuous-flow left ventricular assist device HeartMate II. Circ Heart Fail. 2013;6:517-26.

19. Klovaite J, Gustafsson F, Mortensen SA, et al. Severely impaired von Willebrand factor-dependent platelet aggregation in patients with a continuous-flow left ventricular assist device (HeartMate II). J Am Coll Cardiol. 2009;53:2162-7.

20. Aggarwal A, Raghuvir R, Eryazici P, et al. The development of aortic insufficiency in continuous-flow left ventricular assist device-supported patients. Ann Thorac Surg. 2013;95:493-8.

21. Toda K, Fujita T, Domae K, et al. Late aortic insufficiency related to poor prognosis during left ventricular assist device support. Ann Thorac Surg. 2011;92: 929-34.

22. Goda A, Takayama H, Pak SW, et al. Aortic valve procedures at the time of ventricular assist device placement. Ann Thorac Surg. 2011;91:750-4.

23. Piacentino V 3rd, Ganapathi AM, Stafford-Smith M, et al. Utility of concomitant tricuspid valve procedures for patients undergoing implantation of a continuousflow left ventricular device. J Thorac Cardiovasc Surg. 2012;144:1217-21.

24. Garan AR, Yuzefpolskaya M, Colombo PC, et al. Ventricular arrhythmias and implantable cardioverter-defibrillator therapy in patients with continuous-flow left ventricular assist devices: need for primary prevention? J Am Coll Cardiol. 2013;61:2542-50.

25. Mulloy DP, Bhamidipati CM, Stone ML, et al. Cryoablation during left ventricular assist device implantation reduces postoperative ventricular tachyarrhythmias. J Thorac Cardiovasc Surg. 2013;145:1207-13.

26. Moazami N, Milano CA, John R, et al. Pump replacement for left ventricular assist device failure can be done safely and is associated with low mortality. Ann Thorac Surg. 2013;95:500-5.

27. Uriel N, Morrison KA, Garan AR, et al. Development of a novel echocardiography ramp test for speed optimization and diagnosis of device thrombosis in continuous-flow left ventricular assist devices: the Columbia ramp study. J Am Coll Cardiol. 2012;60:1764-75.

28. Ota T, Yerebakan H, Akashi H, et al. Continuous flow left ventricular assist device exchange: clinical outcomes. J Heart Lung Transplant. 2014;33:65-70.

29. Russell SD, Rogers JG, Milano CA, et al. Renal and hepatic function improve in advanced heart failure patients during continuous-flow support with the HeartMate II left ventricular assist device. Circulation. 2009;120:2352-7.

30. Radovancevic B, Vrtovec B, de Kort E, et al. End-organ function in patients on long-term circulatory support with continuous- or pulsatile-flow assist devices. J Heart Lung Transplant. 2007;26:815-8.

31. Stehlik J, Edwards LB, Kucheryavaya AY, et al. The registry of the international society for heart and lung transplantation: 29th official adult heart transplant report-2012. J Heart Lung Transplant. 2012;31:1052-64.

32. Teuteberg JJ, Stewart GC, Jessup M, et al. Implant strategies change over time and impact outcomes. Insights from the INTERMACS (Interagency Registry for Mechanically Assisted Circulatory Support). JACC Heart Fail. 2013;1: 369-78. 